\title{
GENERALIZED THERMAL INSTABILITY CRITERION OF BLACK HOLE ACCRETION DISKS円
}

\author{
WU Xue-bing ${ }^{1,2}$
}

1. Beijing Astronomical Observatory, Chinese Academy of Sciences, Beijing 100080, China

2. Institute of Theoretical Physics, Chinese Academy of Sciences, Beijing 100080, China

\begin{abstract}
The conventional thermal instability criterion can not be applied to the advectiondominated accretion disks around black holes where the radiative cooling is insufficient to balance the viscous heating. The surface density change associated with the temperature perturbations, which was usually neglected in deriving the conventional criterion, was recently shown to be much significant in the advection-dominated disks. Considering both advection and surface density change, I suggested a generalized thermal instability criterion. By applying it to the optically thin and optically thick advection-dominated disks, I found that the former one is thermally stable and the latter one is thermally unstable against short wavelength perturbations, which agrees well to those found recently by both analytic and quantitative stability analyses.
\end{abstract}

PACS: 97.10.Gz; 97.60.Lf

Accretion disk around black holes can provide a large amounts of released energy and is thus believed to be the source of high energy emission of Galactic black hole X-ray binaries and active galactic nuclei ${ }^{1}$. The stability properties of accretion disks are very important because a global violently unstable disk may not exist in nature and some instabilities restricted in a certain region of the disk may contribute to the observed light variations in many active astronomical systems. Shortly after the construction of the standard model of an optically thick, geometrically thin accretion disk ${ }^{2}$, the inner region of such a disk was found to be both thermally and secularly unstable ${ }^{3,4}$. The hot optically thin disk model, proposed by Shapiro, Lightman \& Eardley ${ }^{5}$ to explain the hard X-ray spectra of black hole X-ray binary Cyg X-1, was also proved to be thermally unstable ${ }^{6}$. The general stability criteria of accretion disks were derived by Piran ${ }^{7}$ who considered various kinds of viscosity laws and cooling processes. These criteria can be used as an a priori check if some choice of cooling mechanisms gives rise to a stable disk even before the complete disk structure equations are solved. The thermal instability criterion can be expressed as ${ }^{8}$ :

$$
\left(\frac{\partial \ln Q^{-}}{\partial \ln T}\right)_{\Sigma}<\left(\frac{\partial \ln Q^{+}}{\partial \ln T}\right)_{\Sigma}
$$

where $Q^{+}, Q^{-}$and $\mathrm{T}$ are the viscous heating rate, radiative cooling rate and temperature. $\Sigma$ is the surface density defined as $\Sigma=\rho H$ where $\rho$ and $H$ are the density and disk height respectively. Such a criterion is identical to that appeared in Piran ${ }^{7}$ if we relace $T$ with $H$. The thermal instability can be well understood according to this criterion. Considering a

\footnotetext{
${ }^{1}$ Supported by the Postdoctoral Science Foundation of China
} 
small temperature increase and assuming that the surface density does not change within the thermal time scale, if the viscous heating rate increases more rapidly than the radiative cooling rate does (as indicated in inequality (1)), it will lead to a further increase of temperature and the thermal instability sets in. If the standard viscosity law is adopted, Piran has pointed out that the inner region of accretion disks is always thermally unstable no matter what kind of cooling mechanism is involved ${ }^{7}$.

However, the thermal instability criterion we mentioned above can not be applied directly to the advection-dominated disks. In the advection-dominated disk models, the radiative cooling is inefficient and most of the energy generated by viscous heating is transfered to increase the entropy of accretion flow and then is advected inwards onto the black hole ${ }^{9,10,11}$. To derive a criterion for the thermal instability of advection-dominated disks, we should consider the advected energy in the energy conversation equation. Moreover, the surface density change associated with the temperature perturbations, which used to be negligible in previous stability analyses of the geometrically thin disks, should be considered in deriving a thermal instability criterion of advection-dominated disks. Some recent works on the stability of advection-dominated disks have indicated that the surface density change is much significant in these disks comparing to the minor pressure change ${ }^{12,13,14}$. In this letter, I will present a generalized thermal instability criterion by considering both advection ad surface density change.

In deriving the conventional thermal instability criterion, the energy balance between heating and cooling was assumed in the unperturbed case. However, in an advection-dominated disk, this balance is realized among viscous heating, radiative cooling and advection (for simplicity, the effects of magnetic field and thermal diffusion are not included in the present study). That is,

$$
Q^{+}=Q^{-}+Q^{a d}
$$

where $Q^{+}, Q^{-}$and $Q^{a d}$ are the viscous heating rate, radiative cooling rate and the energy advection rate respectively. The crucial point of the thermal stability of accretion disks is to examine whether or not this balance is still held if the temperature perturbations are involved. To do this examination, we first write some relative energy changes as follows:

$$
\frac{\delta Q^{+}}{Q^{+}}=x_{1} \frac{\delta T}{T}+y_{1} \frac{\delta \Sigma}{\Sigma}, \quad \frac{\delta Q^{-}}{Q^{-}}=x_{2} \frac{\delta T}{T}+y_{2} \frac{\delta \Sigma}{\Sigma}, \quad \frac{\delta Q^{a d}}{Q^{a d}}=x_{3} \frac{\delta T}{T}+y_{3} \frac{\delta \Sigma}{\Sigma}
$$

where the relative changes of $Q^{+}, Q^{-}$and $Q^{a d}$ are expressed as the functions of temperature and surface density. $x_{i}$ and $y_{i}(i=1,2,3)$ can be defined as:

$$
\begin{aligned}
x_{1}=\left(\frac{\partial \ln Q^{+}}{\partial \ln T}\right)_{\Sigma}, \quad y_{1} & =\left(\frac{\partial \ln Q^{+}}{\partial \ln \Sigma}\right)_{T} ; \quad x_{2}=\left(\frac{\partial \ln Q^{-}}{\partial \ln T}\right)_{\Sigma}, \quad y_{2}=\left(\frac{\partial \ln Q^{-}}{\partial \ln \Sigma}\right)_{T} \\
x_{3} & =\left(\frac{\partial \ln Q^{a d}}{\partial \ln T}\right)_{\Sigma}, \quad y_{3}=\left(\frac{\partial \ln Q^{a d}}{\partial \ln \Sigma}\right)_{T}
\end{aligned}
$$

If we also define $q=Q^{a d} / Q^{+}$and $Q^{-}=(1-q) Q^{+}$, we can obtain:

$$
\frac{\delta Q^{+}-\delta Q^{-}-\delta Q^{a d}}{Q^{+}}=\left[\left(x_{1}-x_{3}\right)-(1-q)\left(x_{2}-x_{3}\right)\right] \frac{\delta T}{T}+\left[\left(y_{1}-y_{3}\right)-(1-q)\left(y_{2}-y_{3}\right)\right] \frac{\delta \Sigma}{\Sigma}
$$


The thermal instability will arise if the left side of above equation is positive, which means that with a small increase of temperature the viscous heating rate grows more fast than the sum of radiative cooling rate and energy advection rate does. Therefore, a thermal instability criterion including the advection term and the surface density change will be:

$$
x_{1}-(1-q) x_{2}-q x_{3}>-\delta_{\Sigma T}\left[y_{1}-(1-q) y_{2}-q y_{3}\right]
$$

where $\delta_{\Sigma T} \equiv \frac{\delta \Sigma}{\Sigma} / \frac{\delta T}{T}$, which describes the relative changes of surface density to the temperature perturbations. This criterion can be regarded as the generalization of the conventional criterion (inequality (1)). If we keep the surface density as a constant and take $q$ as zero, it will be the identical to the inequality (1). Because the advection term and the surface density change are considered in our new criterion, we can use it now to check the thermal instability of advection-dominated disks.

For an optically thin disk, the pressure is dominated by gas and the opacity is dominated by electron scattering ${ }^{10}$. We assume that radiative cooling is provided by thermal bremsstrahlung with emissivity as

$$
Q^{-}=1.24 \times 10^{21} \mathrm{H \rho}^{2} \mathrm{~T}^{1 / 2} \mathrm{ergss}^{-1} \mathrm{~cm}^{-2} .
$$

The viscous heating rate is given by the standard formula

$$
Q^{+}=\Sigma \nu\left(r \frac{\partial \Omega}{\partial r}\right)^{2}
$$

Where $\nu$ is the viscosity parameter and $\Omega$ is the angular velocity. The advection cooling rate is taken as

$$
Q^{a d}=C_{v}\left[\Sigma V_{r} \frac{\partial T}{\partial r}-\left(\Gamma_{3}-1\right) T V_{r} \frac{\partial \Sigma}{\partial r}\right]
$$

where $C_{v}=3 c_{s}^{2} / 2 T, \Gamma_{3}=5 / 3, V_{r}$ is the radial velocity and $c_{s}$ is the local sound speed. Considering $p=K \rho T, H=c_{s} / \Omega_{k}, c_{s}^{2}=p / \rho, \nu=\alpha c_{s} H$ and $V_{r} \sim \nu / r$, we have $Q^{+} \propto \Sigma T$, $Q^{-} \propto \Sigma^{2}$ and $Q^{a d} \propto \Sigma T^{2}[s(T)-s(\Sigma)]$. Here $s(T) \equiv \frac{d l n T}{d l n r}$ and $s(\Sigma) \equiv \frac{d l n \Sigma}{d l n r}$ and we assume them to be constant, which is valid if $T$ and $\Sigma$ are the power law functions of $r$ as indicated, for example, by the self similar solutions ${ }^{9}$. Therefore, for an optically thin disk we have $x_{1}=1, y_{1}=1, x_{2}=0, y_{2}=2, x_{3}=2$ and $y_{3}=1$. Taking these values into inequality (6), we can obtain a criterion for thermal instability of an optically thin disk, that is

$$
\delta_{\Sigma T}<1-\frac{q}{1-q}
$$

If the optically thin disk is radiative cooling dominated, which means $q \sim 0$, above inequality requires $\delta_{\Sigma T}<1$. In the long perturbation wavelength case, there is no appreciable surface density change so we have $\delta_{\Sigma T} \sim 0$. In the short perturbation wavelength case, a recent study by $\mathrm{Wu}^{14}$ showed $-1<\delta_{\Sigma T}<0$. Therefore, our criterion proves again that an optically thin disk is thermally unstable if it is radiative cooling dominated. On the other hand, if the optically thin disk is advection-dominated, which means $q \sim 1$, inequality (10) requires $\delta_{\Sigma T}<-\infty$. Since we always have $-1<\delta_{\Sigma T}<0$ for an optically thin, advection-dominated disk, obviously this inequality means that the disk is thermally stable.

If the optically thin disk is two-temperature one, the radiative cooling may be dominated by Comptonization through the loss of energy of electrons. Then the radiative cooling takes the form of ${ }^{5}$ :

$$
Q_{-}=\left(4 k T_{e} / m_{e} c^{2}\right) \rho H \kappa_{e s} U_{r} c
$$


where $m_{e}, T_{e}$ are the mass and temperature of electron, $\kappa_{e s}=0.4 \mathrm{~cm}^{2} \mathrm{~g}^{-1}$ is the electron scattering opacity. $U_{r}$ is the radiation energy density of soft photons, which we assume, for simplicity, does not change on time scale shorter compared to $\Omega^{-1}$. Because the ions and electrons are coupled by collisional energy exchange, the loss of energy of electrons can be balanced by the energy capture from ions. The exchange rate is

$$
Q_{i-}=(3 / 2) \rho H \nu_{E} k\left(T_{i}-T_{e}\right) / m_{p}
$$

where $\nu_{E}$ is the electron-ion coupling rate, and can be approximated by $\nu_{E}=2.4 \times 10^{21} \ln \Lambda \rho T_{e}{ }^{-3 / 2}$ where the Coulomb logarithm $\ln \Lambda$ is about 15. The ion temperature $T_{i}$ is usually one or two orders higher than the electron temperature $T_{e}$. Taking $Q_{-}=Q_{i-}$ and $T_{i}>>T_{e}$, we can get $Q_{-} \propto \Sigma^{7 / 5} T_{i}^{1 / 5}$. Then we have $x_{2}=1 / 5$ and $y_{2}=7 / 5$, then the thermal instability criterion now becomes:

$$
\delta_{\Sigma T}<2-\frac{5 q}{2(1-q)}
$$

Similar as we discussed above, this inequality clearly shows that a radiative cooling dominated two-temperature disk is thermally unstable and an advection-dominated two-temperature disk is thermally stable. A more recent quantitative stability analysis also reaches the same conclusion ${ }^{15}$. Moreover, from inequalities (10) and (13), we note that in the long wavelength perturbation case where $\delta_{\Sigma T} \sim 0$, the bremsstrahlung cooling disk and the two temperature disk may become thermally stable when $q>\frac{1}{2}$ and $q>\frac{4}{9}$, respectively.

For an optically thick accretion disk, if it is dominated by radiation pressure and electron scattering, we can write the viscous heating rate as $Q^{+}=\Sigma \nu\left(r \frac{\partial \Omega}{\partial r}\right)^{2} \propto \frac{T^{8}}{\Sigma}$ and the radiative cooling rate as $Q^{-}=\frac{4 a c T^{4}}{3 \kappa \Sigma} \propto \frac{T^{4}}{\Sigma}$ where $\kappa$ is the opacity dominated by electron scattering. The advection cooling rate, which is described by equation (9), can be expressed as $Q^{a d} \propto$ $\frac{T^{16}}{\Sigma^{3}}[s(T)-s(\Sigma)]$ for an optically thick disk. Therefore, we have $x_{1}=8, y_{1}=-1, x_{2}=4$, $y_{2}=-1, x_{3}=16$ and $y_{3}=-3$. The criterion for thermal instability of optically thick disks can be written as:

$$
\delta_{\Sigma T}>6-\frac{2}{q}
$$

If the otically thick disk is radiative cooling dominated, above inequality becomes $\delta_{\Sigma T}>$ $-\infty$, which is satisfied without any doubt. Therefore, this indicates that the optically thick, radiation pressure and radiative cooling dominated disk is thermally unstable, which was first proved by a linear study of Shakura \& Sunyaev ${ }^{3}$. If the optically thick disk is advectiondominated, the thermal instability criterion requires $\delta_{\Sigma T}>4$. A recent study of $\mathrm{Wu}{ }^{14}$ pointed out that for an optically thick, advection-dominated disk we have $\delta_{\Sigma T} \sim 8$ in the short wavelength perturbation case, which is the result of the fact that there is no appreciable vertical integrated pressure change associated with the temperature perturbations. Therefore we still obtain that the optically thick, advection-dominated disk is thermally unstable, even if the effects of thermal diffusion and magnetic fields are not included.

In addition, we note that in the long wavelength perturbation limit where we have $\delta_{\Sigma T} \sim 0$, the optically thick disk may become thermally stable when $q>\frac{1}{3}$. This also agrees well with the implication of the turning point from the radiative cooling dominated branch to the advection-dominated branch in the thermal equilibrium curve ${ }^{10}$.

The generalized criterion can be applied to investigate the thermal stability properties of both radiative cooling dominated disks and advection-dominated disks. The results qualitatively agree well to those obtained by some recent detailed stability analyses, which shows 
that an optically thin advection-dominated disk is thermally stable while an optically thick advection-dominated disk is thermally unstable against the short wavelength perturbations 12,13. Moreover, without solving the detailed disk structure, our new criterion can be used to check whether ot not the disk is thermally stable when the advection effect and the different cooling mechanism are involved.

We note that our new criterion depends on the relative surface density change $\delta_{\Sigma T}$, which is a function of the perturbation wavelength and other disk parameters such as viscosity, Mach number, disk height and advection term ${ }^{14}$. In the limit of short or long wavelength perturbations, we can easily obtain the thermal stability properties of the disks from the criterion by adopting the known values of $\delta_{\Sigma T}$. However, in the case of intermediate perturbation wavelength, we have to obtain $\delta_{\Sigma T}$ first by numerically solving the perturbed equations before we adopt the new criterion. In this sense, we think that the generalized thermal instability criterion suggested in this letter is only appropriate in the short and long perturbation wavelength limits and the quantitative stability analyses of accretion disks are still needed for the case of intermediate perturbation wavelength.

I thank Dr. Xingming Chen at Lick Observatory for helpful discussions.

\section{REFERENCES}

1. J.E. Pringle, Ann. Rev. Astro. Astrophy., 19(1981), 137

2. N.I. Shakura, R.A. Sunyaev, Astro. Astrophy., 24(1973), 337

3. N.I. Shakura, R.A. Sunyaev, Mon. Not. Royal. Astro. Soc., 175(1976), 613

4. A.P. Lightman, D.N. Eardley, Astrophy. J., 187(1974), L1

5. S.L. Shapiro, A.P. Lightman, D.N Eardley, Astrophy. J., 204(1976), 187

6. J.E. Pringle, Mon. Not. Royal. Astro. Soc., 177(1976), 65

7. T. Piran, Astrophy. J., 221(1978), 652

8. J. Frank, A.R. King, D.J. Raine, Accretion Power in Astrophysics (2nd edition), Cambridge University Press (1992)

9. R. Narayan, I.Yi, Astrophy. J., 428(1994), L13

10. M.A. Abramowicz, et al. , Astrophy. J., 438(1995), L37

11. X. Chen, et al. , Astrophy. J., 443(1995), L61

12. S. Kato, M.A. Abramowicz, X. Chen, Pub. Astro. Soc. Japan, 48(1996), 67

13. X.B. Wu, Q.B. Li, Astrophy. J., 469(1996), 776

14. X.B. Wu, Astrophy. J., 489(1997), 222

15. X.B. Wu, Mon. Not. Royal. Astro. Soc., 292(1997), 113 